\title{
Emerging Role of Immune Therapy in HCC
}

\author{
Stacey M. Stein, $\mathrm{MD}^{1}{ }^{10}$ \\ ${ }^{1}$ Department of Medicine, Yale School of Medicine, New Haven, \\ Connecticut
}

Dig Dis Interv 2021;5:277-282.

\begin{abstract}
Address for correspondence Stacey M. Stein, MD, Department of Medicine, Yale School of Medicine, 333 Cedar St, New Haven, CT 06520 (e-mail: Stacey.stein@yale.edu).
\end{abstract}

\begin{abstract}
Keywords

- advanced HCC

- immune therapy combinations
\end{abstract}

Hepatocellular cancer (HCC) remains a malignancy with a large unmet need in advanced disease. The incidence rates have been rising over the last three decades and are expected to continue to increase. ${ }^{1}$ Common etiologies of cirrhosis causing HCC remain hepatitis $B$, hepatitis C, and alcoholic liver disease, with nonalcoholic fatty liver disease on the rise. The presence of cirrhosis in most patients with this cancer also complicates treatment options and impacts patient survival.

While early disease may be treated with curative options including surgical resection, ablation, or transplantation, more advanced disease does not have curative intent options. Patients with intermediate disease are often treated with locoregional therapies and patients with advanced disease (including metastatic disease, macro vascular invasion, failure of local therapy) are usually candidates for systemic therapy. Because of the poor survival of patients with decompensated cirrhosis, all trials cited in this review restricted enrollment to patients with Child-Pugh A disease and some studies have also included patients with B7 disease.

received

August 9, 2020 accepted after revision December 1, 2020
Issue Theme Non-Hepatobiliary

Foregut, Midgut, Colorectal Surgery, and Metabolic Surgery; Guest Editors, A. Daniel Guerron, MD, FACS, FASMBS and Kunoor Jain-Spangler, MD

\section{TKIs and VEGF Inhibition in HCC}

Sorafenib is a tyrosine kinase inhibitor (TKI) that acts on multiple targets including the platelet-derived growth factor receptor (PDGFR), RAF kinases, and the vascular endothelial growth factor (VEGF). Sorafenib was US Food and Drug Administration (FDA) approved in 2007 based on results from the SHARP study, which was a randomized study of sorafenib or placebo. ${ }^{2}$ This was the first study in HCC that showed a survival benefit with systemic therapy with an increase in overall survival (OS) from 7.9 to 10.7 months and an overall response rate (ORR) of $2.2 \%$.

Subsequently, two additional TKIs were approved for use as a second-line therapy for patients with advanced HCC, regorafenib, and cabozantinib. The studies for both drugs enrolled patients who had previously received sorafenib and had a Child-Pugh A score. While both drugs are in the same category of TKIs, they have different targets; regorafenib targets include RET, VEGFR 1 to 3 , KIT, and PDGRF- $\alpha$, while cabozantinib targets include EGFR 1 to 3, MET, and AXL. In the RESOURCE study, ${ }^{3} 573$ patients enrolled and were $\begin{array}{ll}\text { (c) 2021. Thieme. All rights reserved. } & \text { DOI https://doi.org/ } \\ \text { Thieme Medical Publishers, Inc., } & 10.1055 / \mathrm{s}-0041-1722932 . \\ 333 \text { Seventh Avenue, 18th Floor, } & \text { ISSN 2472-8721. }\end{array}$ 
randomized $2: 1$ to regorafenib or placebo. OS, the primary end point of the study, improved from 7.8 months on placebo to 10.6 on regorafenib (hazard ratio [HR]: 0.63; 95\% confidence interval $[\mathrm{CI}]:$ 9.1-12.1). The ORR improved from $4 \%$ on placebo to $11 \%$ on regorafenib.

The CELESTIAL study ${ }^{4}$ enrolled a total of 707 patients who were randomized $2: 1$ to receive cabozantinib or placebo. The study met its primary end point of improvement in OS, from 8.0 months with placebo to 10.2 months with cabozantinib (HR for death, $0.76 ; 95 \% \mathrm{CI}: 0.63$ to $0.92 ; p=0.005$ ). The ORR, a secondary end point of the study, was less than $1 \%$ in the placebo arm and $4 \%$ in the cabozantinib arm $(p=0.009)$. Based on these studies, the FDA approved regorafenib in 2017 and cabozantinib in 2019.

Another TKI, lenvatinib, was compared with sorafenib in the phase III REFLECT study, a first-line study that was powered to determine whether lenvatinib was noninferior or superior to sorafenib in patients with advanced HCC and Child-Pugh A liver disease. ${ }^{5}$ The OS was 13.6 months on the lenvatinib arm and 12.3 months on the sorafenib arm, which demonstrated that lenvatinib was noninferior but not superior to sorafenib and led to its FDA approval in 2018. Lenvatinib inhibits VEGF receptors 1 to 3, FGF receptors 1 to 4 , PDGF receptor $\alpha$, RET, and KIT. The ORR was $18.8 \%$ that was significantly better than ORR of sorafenib (6.6\%), making this a preferred first-line TKI treatment.

Ramucirumab, an anti-VEGF antibody, was examined in a nonselected advanced HCC patient population (The REACH study $)^{6}$ that did not meet its primary end point of improvement in OS. The REACH-2 study ${ }^{7}$ then focused on a subset of patients with an $\alpha$-fetal protein (AFP) level greater than 400 and randomized patients with Child-Pugh A liver function to ramucirumab $8 \mathrm{mg} / \mathrm{kg}$ plus best supportive care (BSC) or placebo plus BSC. There was a survival benefit seen in the patients with AFP over 400 with median OS of 8.5 versus 7.3 months. The ORR was $4.6 \%$. There was an increase in proteinuria and hypertension in the ramucirumab arm, and a $7 \%$ incidence of gastrointestinal hemorrhage, including one death, occurred due to esophageal varies hemorrhage. In 2019, ramucirumab was approved for patients with prior sorafenib treatment who have an AFP $>400 \mathrm{ng} / \mathrm{mL}$.

Prior to the REACH studies, bevacizumab, also an anti-VEGF antibody, was examined in advanced HCC in a small phase 2 study of 46 patients. $^{8}$ The ORR was $13 \%$ (6 patients) and the median progression-free survival (mPFS) was 6.9 months. There were grade 3 or higher hemorrhages in $11 \%$ of patients including one patient death from a variceal bleed. While there have been other studies that have incorporated bevacizumab into HCC treatment, bevacizumab was not submitted for approval to the FDA based on single agent data.

\section{Background for Immune Therapy in HCC}

There is a complex interplay between cancer and the immune system, especially in HCC where cancer arises in an immune organ and underlying causes of cirrhosis cause chronic inflammation. ${ }^{9,10}$ Cells specific to the liver, including Kupffer cells, liver sinusoidal endothelial cells, and hepatic stellate cells, modulate immune responses to pathogens and cancer cells. The liver requires some tolerance to the high level of antigens it is exposed to from the gastrointestinal tract; however, as changes of fibrosis and cirrhosis occur in livers that are damaged by hepatitis $B$ and $C$ viral infections, alcohol exposure, and other chronic inflammatory changes, there are changes to the immunogenicity of the liver. The HCC microenvironment is characterized by dysfunction of different immune components. There can be increased tolerance of pathogens, contributing to a hypoxic environment, which can cause VEGF upregulation. There is increased expression of PD-L1 in tumor biopsies as compared with biopsies of liver background. Therefore, there was strong preclinical rational for examining the roles of PD-1 and PD-L1 inhibition and for immune therapy combinations in HCC.

\section{Single-Agent PD-1 Data}

Nivolumab

The use of checkpoint inhibitors has shown great benefit in several cancer types and they were eventually examined in HCC. There was early concern that viral reactivation could occur with checkpoint inhibition and early trials with nivolumab and pembrolizumab enrolled patients with hepatitis $B$, hepatitis $C$, and nonviral etiologies to examine whether efficacy and safety profile varied by different causes of cirrhosis.

Nivolumab, a fully human anti-IgG4 monoclonal antibody that disrupts PD-1 immune checkpoint signaling, was first examined in HCC as part of the phase $1 / 2$ study CheckMate $040 .{ }^{11}$ Patients enrolled had advanced HCC and Child-Pugh scores of A or B7. They either had progression of disease on sorafenib, were intolerant of sorafenib, or chose to enroll on the study without prior sorafenib treatment. The analysis included 48 patients in a dose-escalation part and 214 patients in the expansion cohort. In the dose-escalation cohort, patients received nivolumab intravenous (IV) every 2 weeks at doses from 0.1 to $10 \mathrm{mg} / \mathrm{kg}$ and although a maximally tolerated dose was not reached, the expansion cohort dose was chosen to be $3 \mathrm{mg} / \mathrm{kg}$ based on data from other nivolumab studies.

In the dose-expansion phase, patients were enrolled into four cohorts: sorafenib naïve or intolerant, sorafenib progressor without viral hepatitis, HCV infected, and HBV infected. Primary end points were safety and tolerability for the escalation phase and objective response rate for the expansion phase.

The ORR was $15 \%$ in the dose-escalation phase, with four partial responses (PRs) and three complete responses (CRs) and responses did not correlate depending on viral status. The median time to progression was 3.4 months $(95 \% \mathrm{CI}$ : 1.6-6.9). The median duration of response (DOR) was 17 months (95\% CI: 6-24). The mOS for patients in the dose-escalation phase was 15.0 months (95\% CI: 9.6-20.2). Grade 3 and 4 adverse events (AEs) occurred in 12 (25\%) of patients including adrenal insufficiency (1), diarrhea (1), hepatitis (2), infusion hypersensitivity (1), and acute kidney injury (1). There were no treatment-related deaths. There was no association between response and PD-L1 expression. Based on this data, in 2017, the FDA gave accelerated 
approval for the use of nivolumab for patients with advanced HCC who had previously received sorafenib.

The phase 3 CheckMate 459 study randomized 743 patients 1:1 with advanced HCC to nivolumab or sorafenib in the first line. ${ }^{12}$ Median OS, the primary end point, was longer on the nivolumab arm (16.4 vs. 14.7 months, HR: 0.85 ; $95 \%$ CI: $0.72-1.02$ ), which was not statistically significant $(p=0.0419)$. However, $47 \%$ of patients in the sorafenib arm went on to receive another systemic therapy, many of them receiving subsequent immune therapy. ORR, a secondary end point, was $14.3 \%$ in the nivolumab arm. There were no new safety signals.

\section{Pembrolizumab}

Pembrolizumab was initially examined in HCC in the singlearm phase 2 KEYNOTE-224 study. ${ }^{13}$ Patients with ChildPugh A disease and advanced HCC received pembrolizumab $200 \mathrm{mg}$ every 3 weeks for up to 2 years. The primary end point of response rate was met with 18 of 104 patients (17\%) having an objective response, 1 of the 18 was a CR. AEs included serious events in 16 (15\%) of patients including one death from ulcerative esophagitis. There were no cases of viral flares. There was some correlation between PD-L1 expression and response, using a combined score of tumor and microenvironment PD-L1 expression. Based on these data, the FDA gave accelerated approval for pembrolizumab in second-line therapy in advanced HCC in 2018 and the phase 3 KEYNOTE-240 study opened.

The KEYNOTE-240 study was a randomized, double-blind, phase 3 , second-line study of pembrolizumab versus placebo after sorafenib treatment. ${ }^{14}$ Median OS was 13.8 months for the pembrolizumab arm and 10.6 months for the placebo arm (HR: 0.781; 95\% CI: 0.611-0.998; $p=0.0238$ ); however, since OS and PFS were coprimary end points of the study the statistical end point of the study included a $p$-value of less than the standard 0.05 and was not met. Median PFS for pembrolizumab was 3.0 months compared with 2.8 months for the placebo. (HR: $0.718 ; 95 \% \mathrm{CI}: 0.570-0.904 ; p=0.0022$ ).

The ORR was similar to the phase 1 study of pembrolizumab 18.3 versus $4.4 \%$ for placebo. The median DOR was 13.8 months. The association between PD-L1 score and clinical response noted in the KEYNOTE-224 study was not replicated in the 240 study.

\section{Summary of Single-Agent PD-1 Data}

The randomized data for single-agent PD-1 inhibition was disappointing after early data showed response rates of 15 to $17 \%$. Potential factors that contributed to negative data in these two randomized studies were the statistical designs of the studies and most importantly, the lack of a biomarker for selecting patients. While AFP has possibly selected a more aggressive disease course in some trials, it has not correlated with response to any treatment other than ramucirumab. Also, there has been no consistent signal in PD-L1 expression and response to treatment across these studies. Therefore, without any selection criteria for these patients, there was no statistically significant benefit as compared with sorafenib or
BSC. These two negative randomized studies highlight the need for more effective therapy combinations.

\section{Combination Immune Therapy}

\section{Atezolizumab and Bevacizumab}

The combination of atezolizumab and bevacizumab was examined in patients with advanced HCC as one arm on the phase $1 \mathrm{GO} 30140$ study. ${ }^{15}$ Eligible patients had unresectable HCC not amenable to curative treatment, with measurable disease by RECIST v 1.1, no prior systemic therapy, and an ECOG PS of 0 or 1 . In addition, all patients had an esophagogastric endoscopy within 6 months of treatment and any varices had to have been treated per local protocol prior to enrollment.

Patients received atezolizumab $1200 \mathrm{mg}$ and bevacizumab $15 \mathrm{mg} / \mathrm{kg}$ IV every 3 weeks. A total of 104 patients were enrolled and the confirmed ORR by independent review was $36 \%$ (37 patients) (95\% CI: $26-46), 12$ (12\%) of patients had a CR. At the time of data cutoff, 28 (76\%) of 37 responders had an ongoing response and the median DOR was not reached. Response did not correlate with PD-L1 status. The most common grade 3 to 4 AEs were hypertension (13\%) and proteinuria (7\%). Serious bleeding events occurred in four patients-two with upper gastrointestinal hemorrhage and two with esophageal varices hemorrhage.

Later, a second HCC arm of the study was added, which randomized 119 patients to the combination or atezolizumab alone. PFS, the primary end point of this randomized arm, was significantly improved in the combination arm, confirming the need for the combination over atezolizumab alone.

Based on these positive data, the IMbrave 150 study was designed to examine the role of the combination of atezolizumab and bevacizumab versus sorafenib. ${ }^{16}$ A total of 501 patients were randomized 2:1. Patients received atezolizumab $1200 \mathrm{mg}$ IV on day 1 of each 21-day cycle along with bevacizumab $15 \mathrm{mg} / \mathrm{kg}$. Oral sorafenib was administered at the standard full dose of $400 \mathrm{mg}$ twice daily on days 1 to 21 of a 21-day cycle. All patients were required to have an endoscopy within 6 months and varices had to have been treated prior to starting therapy.

There were two primary end points, OS and independent reviewed PFS. OS increased by $42 \%$ (HR: $0.58 ; 95 \% \mathrm{CI}$ : $0.42-0.78 p=0.0006$ ). PFS increased by $41 \%$ (HR: 0.59 ; $95 \%$ CI: $0.47-0.76 p=0.0001$ ). There were grades 3 to 4 AEs in $56.5 \%$ of patients in the combination arm and $55.1 \%$ on the sorafenib arm. The most common AEs (>20\%) were hypertension, fatigue, and proteinuria with atezolizumab and bevacizumab. There were 9 (5.8\%) grade 5 events on the sorafenib arm, including one peritoneal hemorrhage and 15 (4.6\%) grade 5 events on the atezolizumab and bevacizumab arm including one esophageal varices hemorrhage and one subarachnoid hemorrhage. Median OS was 17.1 months (95\% CI: 13.8-not estimable) with 57 (55\%) of patients alive at the time of the data cutoff.

Patient-reported outcomes were better than sorafenib, as assessed by the European Organization for the Research and Treatment of Cancer Quality-of-Life Questionnaire(EORTCQLQ- 
C30). ${ }^{17}$ Patients experienced less deterioration of quality-of-life on the combination arm, which was a prespecified end point. The median time to quality-of-life deterioration was 3.6 months for patients treated with sorafenib and 11.2 months for patients treated with atezolizumab and bevacizumab (HR: 0.63).

This is the first study since the SHARP study to show a survival benefit in the first-line setting for systemic therapy. The atezolizumab and bevacizumab regimen was approved by the FDA in May 2020 and has changed the landscape for the treatment for advanced HCC.

\section{Combination of PD-L1 and TKIs}

\section{Lenvatinib and Pembrolizumab}

In a phase 1 study, a total of 104 patients received lenvatinib $12 \mathrm{mg}$ ( or $8 \mathrm{mg}$ if under $60 \mathrm{~kg}$ ) orally daily and pembrolizumab $200 \mathrm{mg}$ IV on day 1 of a 21 -day cycle. ${ }^{18}$ The ORR was $36 \%$ (95\% CI: 26.6-46.0\%) per RECISTv 1.1. The median DOR by independent review was 12.6 months (95\% CI: 6.9 months to not estimable) per RECIST v1.1. Median OS was 22 months. AEs reflected the use of a TKI added to immune therapy. Hypertension occurred in $36 \%$ of patients, diarrhea in $35 \%$, fatigue in $30 \%$, decreased appetite in $28 \%$, and hypothyroidism in $25 \%$. Only one patient had a grade $4 \mathrm{AE}$ of leukopenia/neutropenia and three patients had grade 5 events-one with intestinal perforation, one with abnormal hepatic function, and one with acute respiratory failure.

Based on these results, a phase 3 study of the combination of lenvatinib and pembrolizumab versus single-agent lenvatinib in advanced HCC (the LEAP-002 study) is ongoing. ${ }^{19}$ The phase 1 data was submitted to the FDA for potential accelerated approval; however, given recent approval of the atezolizumab and bevacizumab combination, this study was thought to not show evidence of meaningful improvement over available therapies, and did not meet the criteria for accelerated approval. ${ }^{20}$ The results of the LEAP-002 study will help determine the role of the combination in the future.

\section{Cabozantinib and Atezolizumab}

Another anti-PD-L1 antibody and TKI combination under investigation is cabozantinib and atezolizumab in the firstline COSMIC-312 study. ${ }^{21}$ This study plans to enroll a total of 740 patients in a 2:1:1 randomization to cabozantinib $40 \mathrm{mg}$ daily plus atezolizumab $1200 \mathrm{mg}$ IV every 3 weeks, sorafenib $400 \mathrm{mg}$ per os (PO) twice a day, or cabozantinib $60 \mathrm{mg}$ PO daily. Randomization is stratified by disease etiology, region, and presence of extrahepatic disease or macrovascular invasion. Primary end points are PFS and OS of the combination versus sorafenib; the secondary end point is PFS of cabozantinib versus sorafenib.

\section{Combination of PDL-1 and CTLA-4 Antibodies}

There was strong rationale to build on the single-agent data in nivolumab and pembrolizumab to examine combination regimens with anti-CTLA-4 antibodies, another checkpoint inhibitor. ${ }^{22}$ Anti-CTLA- 4 drugs can release inhibition of T cell activation in lymph nodes, thereby creating an additive benefit to PD-1 or PD-L1 inhibition. The data in melanoma has shown an increased survival benefit with the combination of nivolumab and ipilimumab, an anti-CTLA-4 antibody, with an increase in autoimmune-related AEs. ${ }^{23}$

\section{Nivolumab and Ipilimumab}

There was an additional cohort to the CheckMate040 trial that examined the combination of nivolumab $1 \mathrm{mg} / \mathrm{kg}$ with ipilimumab $3 \mathrm{mg} / \mathrm{kg}$ every 3 weeks for 4 doses, and continued with nivolumab $240 \mathrm{mg}$ every 2 weeks until disease progression or unacceptable toxicity. ${ }^{24}$ All of the 49 patients had received prior sorafenib, and the patient population reflected the HCC population: median age was 60 , $88 \%$ were male, $74 \%$ were Asian, $80 \%$ had extrahepatic spread.

Responses were assessed by blinded independent central review using RECIST v1.1. Sixteen (33\%) patients had an objective response and four patients (8\%) had a CR. The DOR ranged from 4.6 to $30.5+$ months. The most common grade 3 or 4 AEs were rash (53\%), pruritis (53\%), musculoskeletal pain (41\%), diarrhea (39\%), cough (37\%), decreased appetite (35\%), fatigue (27\%), pyrexia (27\%), abdominal pain (22\%), headache (22\%), nausea (20\%), hypothyroidism (20\%), dizziness (20\%), and decreased weight (20\%).

The combination of ipilimumab and nivolumab was approved by the FDA in March 2020 for patients with advanced HCC previously treated with sorafenib. In addition, there is another arm of the CheckMate 040 studies that randomized 71 patients to ipilimumab and cabozantinib versus nivolumab, ipiliumumab, and cabozantinib. ${ }^{25}$ Preliminary data has been reported with a mPFS of 5.5 months in the doublet and 6.8 months in the triplet, while survival data continues to be collected. More grade 3 to 4 AEs were reported in the triplet regimen $(71 \%)$ than in the doublet regimen (42\%).

\section{Durvalumab and Tremelimumab}

The HIMALAYA study is a randomized, open-label, multicenter study that assigned patients with advanced HCC to either durvalumab (an anti-PD-L1 antibody), tremelimumab (an anti-CTLA-4 antibody) or the combination of durvalumab plus tremelimumab in two different doses, or sorafenib. ${ }^{26,27}$ The primary end point of the study is OS and secondary end points include PFS and ORR, TTP, DCR, and DOR.

Data for over 300 patients have been presented at several meetings in 2020. The study has had 3 parts, with the last part examining four separate arms: durvalumab alone, tremelimumab alone, tremelimumab $300 \mathrm{mg}$ and durvalumab 1500 mg every 4 weeks for 4 doses followed by durvalumab every 4 weeks or single-agent durvalumab $1500 \mathrm{mg}$ every 4 weeks or tremelimumab $75 \mathrm{mg}$ every 4 weeks. There was also a cohort in the $2 \mathrm{~B}$ portion of the trial that received the combination, but only one dose of tremelimumab at the $300 \mathrm{mg}$ dose.

The median OS was longest in the combination arm with the $300 \mathrm{mg}$ tremelimumab dose, which was 18.7 months, compared with 13.6 months with durvalumab alone, and 15.1 months for tremelimumab alone. The rate of grade $3 / 4$ autoimmune AEs was $\sim 40 \%$ in both combination arms and 
the tremelimumab arm and lower in the durvalumab arm ( 20\%). It will be important to see final data on this trial to determine the most safe and effective dosing strategy for this regimen.

\section{Conclusion}

There has been a deluge of new data for the treatment of advanced HCC over the last few years. Initial studies with PD-1 checkpoint inhibitors showed response rates in the 15 to $18 \%$ range without meeting statistical end points in randomized studies. More recently, with combination therapy of an anti-PDL-1 antibody with either a VEGF inhibitor, a TKI, or an anti- CTLA-4 antibody, response rates are now over $30 \%$.

The atezolizumab and bevacizumab combination is now the standard of care for first-line patients who meet eligibility criteria. An important consideration for this regimen will be the incorporation of variceal screening prior to initiation of treatment, with the goal of mitigating bleeding events. Also, it is not clear which treatment should be used in the second line for patients who receive combination therapy in the first line.

There remain many other unanswered questions including which treatment algorithms are best for patients with Child-Pugh B disease, who frequently are treated with systemic therapy but not represented in clinical trials. In addition, there is no biomarker which predicts response to any particular drug or combination treatment. PDL-1 expression, which has been used to differentiate response to immune therapy in some diseases, has not correlated with response in HCC. This effort has likely been hampered by the low rate of tissue collection in this disease, as diagnosis is still often made by imaging alone.

\section{Future Directions}

Now that we have more effective treatment options for advanced HCC, it will be important to explore the role of these treatments at other stages of disease. The role of adjuvant therapy after resection or curative radiofrequency ablation is being explored through several current studies. In addition, the question of when to shift from locoregional therapy to systemic therapy remains an important open topic that is frequently debated at multidisciplinary tumor boards. The importance of defining the role for combination systemic therapy in the Barcelona Clinic Liver Cancer B population will be addressed in multiple studies for this population. Also, the higher response rates with combination therapies introduce the possibility of more downstaging of patients to curative intent therapy options.

In just the last year, we have approvals for atezolizumab and bevacizumab in the first-line setting and nivolumab and ipilimumab in the second-line setting with multiple ongoing studies that will be resulted in the next year. These studies will help add to our understanding of the role of checkpoint inhibitors as part of combination therapy for HCC and help create treatment algorithms to best treat our patients.

Conflict of Interest

None.

\section{References}

1 Petrick JL, Kelly SP, Altekruse SF, McGlynn KA, Rosenberg PS. Future of hepatocellular carcinoma incidence in the United States Forecast through 2030. J Clin Oncol 2016;34(15):1787-1794

2 Llovet JM, Ricci S, Mazzaferro V, et al;SHARP Investigators Study Group. Sorafenib in advanced hepatocellular carcinoma. N Engl J Med 2008;359(04):378-390

3 Bruix J, Qin S, Merle P, et al;RESORCE Investigators. Regorafenib for patients with hepatocellular carcinoma who progressed on sorafenib treatment (RESORCE): a randomised, double-blind, placebo-controlled, phase 3 trial. Lancet 2017;389(10064):56-66

4 Abou-Alfa GK, Meyer T, Cheng AL, et al. Cabozantinib in patients with advanced and progressing hepatocellular carcinoma. N Engl J Med 2018;379(01):54-63

5 Kudo M, Finn RS, Qin S, et al. Lenvatinib versus sorafenib in firstline treatment of patients with unresectable hepatocellular carcinoma: a randomized phase 3 non- inferiority trial. Lancet. Doi: 10.1016/SO140-6736(18)30207-1

6 Zhu AX, Park JO, Ryoo BY, et al;REACH Trial Investigators. Ramucirumab versus placebo as second-line treatment in patients with advanced hepatocellular carcinoma following first-line therapy with sorafenib (REACH): a randomised, double-blind, multicentre, phase 3 trial. Lancet Oncol 2015;16(07):859-870

7 Zhu AX, Kang YK, Yen CJ, et al;REACH-2 study investigators. Ramucirumab after sorafenib in patients with advanced hepatocellular carcinoma and increased $\alpha$-fetoprotein concentrations (REACH-2): a randomised, double-blind, placebo-controlled, phase 3 trial. Lancet Oncol 2019;20(02):282-296

8 Siegel AB, Cohen EI, Ocean A, et al. Phase II trial evaluating the clinical and biologic effects of bevacizumab in unresectable hepatocellular carcinoma. J Clin Oncol 2008;26(18):2992-2998

9 O'Rourke JM, Sagar VM, Shah T, Shetty S. Carcinogenesis on the background of liver fibrosis: implications for the management of hepatocellular cancer. World J Gastroenterol 2018;24(39): 4436-4447

10 Yarchoan M, Xing D, Luan L, et al. Characterization of the immune microenvironment in hepatocellular carcinoma. Clin Cancer Res 2017;23(23):7333-7339

11 El-Khoueiry AB, Sangro B, Yau T, et al. Nivolumab in patients with advanced hepatocellular carcinoma (CheckMate 040): an openlabel, non-comparative, phase $1 / 2$ dose escalation and expansion trial. Lancet 2017;389(10088):2492-2502

12 Yau T, Park JW, Finn RS, et al. CheckMate 459: a randomized, multicenter phase III study of nivolumab (NIVO) vs sorafenib (SOR) as first-line (1L) treatment in patients (pts) with advanced hepatocellular carcinoma (aHCC). Ann Oncol 2019;30:V874-V875

13 Zhu AX, Finn RS, Edeline J, et al;KEYNOTE-224 investigators. Pembrolizumab in patients with advanced hepatocellular carcinoma previously treated with sorafenib (KEYNOTE-224): a nonrandomised, open-label phase 2 trial. Lancet Oncol 2018;19(07): 940-952

14 Finn RS, Ryoo BY, Merle P, et al;KEYNOTE-240 investigators. Pembrolizumab as second-line therapy in patients with advanced hepatocellular carcinoma in KEYNOTE-240: a randomized, double-blind, phase III trial. J Clin Oncol 2020;38(03):193-202

15 Lee MS, Ryoo BY, Hsu CH, et al;GO30140 investigators. Atezolizumab with or without bevacizumab in unresectable hepatocellular carcinoma (GO30140): an open-label, multicentre, phase 1b study. Lancet Oncol 2020;21(06):808-820 
16 Finn RS, Qin S, Ikeda M, et al;IMbrave150 Investigators. Atezolizumab plus bevacizumab in unresectable hepatocellular carcinoma. N Engl J Med 2020;382(20):1894-1905

17 Galle PR, Finn RS, Qin S, et al. Patient-reported outcomes (PROs) from the phase III IMbrave 150 trial of atezolizumab (atezo) + bevacizumab (bev) vs sorafenib (sor) as first treatment (tx) for patients (pts) with unresectable hepatocellular carcinoma (HCC). Ann Oncol 2019;30(09):AbstractLBA3

18 Zhu AX, Finn RS, Ikeda M, et al. A phase 1b study of lenvatinib plus pembrolizumab in unresectable hepatocellular carcinoma. Presented at: ASCO Virtual Scientific Program. J Clin Oncol 2020; $38: 4519$

19 Lovet JM, Kudo M, Chang AL, et al. Lenvatinib (Len) plus pembrolizumab (Pembro) for the first-line treatment of patients (pts) with advanced hepatocellular carcinoma (HCC): Phase 3 LEAP002 study. J Clin Oncol 2019;37(15):TPS4152-TPS4152

20 Merck and Eisai Receive Complete Response Letter for KEYTRUDA (pembrolizumab) plus LENVIMA (lenvatinib) Combination as First-Line Treatment for Unresectable Hepatocellular Carcinoma News release. Merck and Eisai. July 8, 2020https://bwnews.pr/ 2Z89nmX

21 Kelley RK, W Oliver J, Hazra S, et al. Cabozantinib in combination with atezolizumab versus sorafenib in treatment-naive advanced hepatocellular carcinoma: COSMIC-312 phase III study design. Future Oncol 2020;16(21):1525-1536
22 Kudo M. Scientific rationale for combination immunotherapy of hepatocellular carcinoma with anti-PD-1/PD-L1 and anti-CTLA-4 antibodies. Liver Cancer 2019;8(06):413-426

23 Wolchok JD, Chiarion-Sileni V, Gonzalez R, et al. Overall survival with combined nivolumab and ipilimumab in advanced melanoma. N Engl J Med 2017;377(14):1345-1356

24 Yau T, Kang Y-K, Kim T-Y, et al. Nivolumab (NIVO) + ipilimumab (IPI) combination therapy in patients (pts) with advanced hepatocellular carcinoma (aHCC): Results from CheckMate 040. J Clin Oncol 2019;37(Suppl 15):4012. Doi: 10.1200/JCO.2019.37.15_suppl.4012

25 Yau T, Zagonel V, Santoro A, et al. Nivolumab (NIVO) + ipilimumab (IPI) + cabozantinib (CABO) combination therapy in patients (pts) with advanced hepatocellular carcinoma (aHCC): results from CheckMate 040. J Clin Oncol 2020;38(04):478-478

26 Kelley RK, Sangro B, Harris WP, et al. Efficacy, tolerability, and biologic activity of a novel regimen of tremelimumab in combination with durvalumab for patients with advanced hepatoceullar carcinoma. J Clin Oncol 2020;38(15):4508-4508

27 Kelley RK, Kudo M, Harris W, et al. The novel regimen of tremelimumab in combination with durvalumab provides a favorable safety profile and clinical activity for patients with advanced hepatocellular carcinoma (aHCC). Presented at: ESMO World Congress on Gastrointestinal Cancer 2020. July 1-4, 2020; Virtual. Abstract 0-6 